\title{
Genetic Diversity Analysis of Different Wheat [Triticum aestivum (L.)] Varieties Using SSR Markers
}

\author{
Summy Yadav*, AkdasbanuVijapura, Akanksha Dave, Sneha Shah and ZebaMemon
}

Division of Biological and Life Sciences, School of Arts and Sciences, Ahmedabad University, Ahmedabad 380009, Gujarat, India

*Corresponding author

\begin{abstract}
A B S T R A C T
Keywords

Triticum aestivum,

Genetic diversity,

SSR markers,

Cluster analysis

Article Info

Accepted:

07 January 2019

Available Online:

10 February 2019

Genetic diversity analysis of nine varieties of wheat (Triticum aestivum) was evaluated using 14 SSR markers. A genetic relationship was studied by calculating the genetic distances using an un-weighted pair-group method with arithmetic mean (UPGMA) subprogram of NTSYS-PC software. The cluster analysis shows that the most closely related varieties were V6 (GW1255) and V9 (GW366); V4 (GW11) and V8 (GW273), V1 (GW503) and V3 (GW451) respectively. V7 (GW173) and V3 (GW451) were the most distinct varieties among all the 9 varieties analyzed in this study. The cluster analysis results were further verified by calculation of the significance and correlation using Pearson correlation analysis. From the results, it was concluded that evaluation of genetic diversity and identification of wheat varieties by the Marker Assisted Selection technology is easy and early approach compared to conventional breeding approaches.

\section{Introduction}

Wheat is a cereal grass which is the $3^{\text {rd }}$ most cultivated plant worldwide. It is selfpollinating annual plant, belonging to the family Poaceae (grasses) and genus Triticum (Shewry 2009). Genetic diversity is the primary requirement to initiate a successful breeding programme for the betterment of wheat productivity. The selection of diverse genotypes is the preliminary requisite for molecular breeding of wheat (Raj et al., 2017). Molecular markers have come up as an effective tool for characterization of genetic material. Genetic markers can be used to

specify the genetic differences between various species.

Genetic markers are biological compounds which can be resolved by allelic variations and can be used as experimental labels or probes to track a discrete, tissue, cell, nucleus, chromosomes or genes. There are three major types of genetic markers: (a) Morphological markers (which are also called "classical" or "visible" markers) which are phenotypic traits, (b) Biochemical markers, which are called isozymes, including allelic variants of enzymes, and (c) DNA markers (or molecular markers), which reveals sites of variation in
\end{abstract}


DNA (Raj et al., 2017; Kumar et al., 2016; Kesawat and Das Kumar, 2009).

Among genetic markers, molecular markers are mainly used because of their relative abundance. Molecular markers have been playing a major role in biotechnology and genetics studies during the last few decades(Kesawat and Das Kumar 2009). They have come up as an effective tool for characterization of genetic material. Molecular markers are independent of environmental conditions under which phenotypic studies are carried out (Kesawat and Das Kumar, 2009).

They play an important role in genetic studies and biotechnology by providing new dimension, accuracy, and perfection in the screening of germ-plasm (Kumar et al., 2016). These markers are selectively neutral as they are usually located in non- coding region of DNA (Lateef, 2015). Unlike biochemical and morphological markers, DNA markers are practically unlimited in number and are not affected by environmental factors as well as the developmental stage of the plant. These molecular markers include: (i) hybridization-based markers such as Restriction Fragment Length Polymorphism (RFLP) (ii) PCR-based markers: Random Amplification of Polymorphic DNA (RAPD), Amplified Fragment Length Polymorphism (AFLP) and Microsatellite or Simple Sequence Repeat (SSR) (iii) Sequenced-based Markers: Single Nucleotide Polymorphism (SNP) (Kesawat and Das Kumar, 2009).

Microsatellites or Simple Sequence Repeats (SSRs) are an efficient tool in diversity studies for identifying the degree of genetic similarity. Due to their high rate of polymorphism i.e. high Polymorphic Information Content (PIC), co-dominant character, selective neutrality, distribution across the genome, environment independent characteristics and cost and labor efficiency, SSR markers are suitable for detecting allele frequency within the population and for assessing population structure(Kumar et al., 2016). At present, SSR markers are one of the most effective molecular markers for genetic differentiation within interspecific or intraspecific species. SSR markers have major applications as highly variable and multiallelic PCR based genetic markers as they are ubiquitously spread in eukaryotic genomes (Kesawat and Das Kumar, 2009).

Due to a high degree of polymorphism and easy handling, SSR markers have various applications in crop improvement. Keeping the advantages of SSR markers in consideration, the present research work was carried out to study genetic variation among various wheat varieties using chromosome specific SSR markers and to find genetically most diverse genotypes of wheat which can further be used in hybridization programs to create genetically diverse germ-plasm of local wheat (Kumar et al., 2016; Kesawat and Das Kumar, 2009; Lateef, 2015).

\section{Materials and Methods}

Nine varieties of wheat were procured from GSSC (Gujarat State Seed Corporation Ltd.) and sown in the crop seasons on November 21st in 2017 for studying the genetic diversity using chromosome specific SSR markers.

\section{Genomic DNA isolation, purification and Quantification}

Genomic DNA was isolated using the CTAB method from a small amount of fresh leaf tissue $(5.0 \mathrm{~g})$ from each variety on January $21^{\text {st }}, 2018$ (Saghai-Maroof et al., 1984). Agarose gel electrophoresis $(0.8 \%)$ was used to check quality of genomic DNA. The DNA concentration and quantity was checked by UV spectrophotometer (Jiang, 2013). 


\section{PCR Amplification}

Wheat varieties were screened using 14 SSR markers for molecular characterization and used for genetic diversity (Tomar et al., 2016a). The PCR reaction was carried out in a reaction mixture of $20 \mu \mathrm{l}$ containing $2 \mu \mathrm{l}$ of $10 \mathrm{X}$ assay buffer, $0.5 \mu \mathrm{l}$ of each primer, $2 \mu 1$ of $25 \mathrm{mM} \mathrm{MgCl}_{2}, 100 \mu \mathrm{M}$ dNTPs, $0.5 \mu \mathrm{l}$ of Taq DNA polymerase and template DNA (Table 1). The thermocycling program was optimized at initial denaturation at $95^{\circ} \mathrm{C}$ for 4 minutes followed by 40 cycles of $95^{\circ} \mathrm{C}$ for 1 minute, 1 minute and 20 second at annealing temperature $\left(52-63^{\circ} \mathrm{C}\right), 1$ minute at $72^{\circ} \mathrm{C}$ for extension, a final cycle of $72^{\circ} \mathrm{C}$ for 10 minutes and hold at $4^{\circ} \mathrm{C}$ (Kumar et al., 2016). The amplified product was resolved on $0.8 \%$ agarose gel electrophoresis. Gels were run at $100 \mathrm{~V}$ for 45 minutes. DNA bands were visualized in UV trans-illuminator and gel dock after completion of electrophoresis (Shuaib et al., 2010).

\section{Data analysis}

Frequency of polymorphism between different varieties of wheat for each type of marker was calculated based on the presence (taken as 1) or absence (taken as 0) of bands. The 0/1 matrix was used to calculate similarity genetic distance using an unweighted pair-group method with arithmetic mean (UPGMA) subprogram of software NTSYS-PC (Numerical Taxonomy and Multivariate Analysis System Programme). The resultant distance matrix was employed to construct dendrogram by the Un-weighted Pair- Group Method with Arithmetic Average (UPGMA) subprogram of NTSYS-PC (Tomar et al., 2016b).

\section{Results and Discussion}

The nine varieties selected for present study are Rabi crops and are majorly grown in
Madhya Pradesh, Gujarat and some parts of Rajasthan. LOC 1, developed by Lokbharti Gramvidhyapith, Sanosora, Gujarat and is one of the most preferred cultivar of wheat in Gujarat. GW 273, GW 366 has made major impact in increasing the productivity of wheat in Gujarat. GW 496, GW 503, GW 451, GW 11, GW1255 and GW 173 are the wheat varieties suitable for timely sown and irrigated conditions in Gujarat (Arun Gupta et al., n.d.). All the nine varieties are the major cultivars of wheat in Gujarat and hence these varieties were selected to check the genetic diversities between these varieties and can there be a future scope of breeding between these varieties.

SSR markers are small DNA motifs that are highly distributed and conserved among the genomes of all higher eukaryotes (Liu et al., 2007). Genetic diversity plays an important role in crop improvement and was demonstrated through SSR markers et al., 2007; Al Khanjari et al., 2007). SSRs have become the marker of choice for an array of applications in plants due to extensive genomiccoverage and hypervariable nature (Al Khanjari et al., 2007; Salem et al., n.d.).

\section{Age analysis}

In the present study, 14 SSR primers were used to estimate the genetic polymorphism of wheat varieties and find out the most diverse varieties for future breeding programs. Among 14 primers, GWM 437 marker did not show any amplification(Ijaz and Khan 2009). Among the 13 primers four primers GWM 610, GWM 369, GWM 247, and WMC 048 produced polymorphic bands and remaining 9 primers are monomorphic. A total of 108 bands were produced from 13 primers. In this study, different wheat varieties were separated by AGE electrophoresis based on high and low molecular weight for characterization and evaluation of genetic 
diversity among 9 varieties(Tomar et al., 2016a).

\section{Cluster analysis}

In the present study, 14 Simple Sequence Repeat (SSR) primer sets were used to characterize nine wheat varieties to know about the diverse varieties for future breeding programs to increase wheat production. The allelic diversity data of SSR primer are used to construct a dendrogram by using a cluster, subprogram of the same software, which shows the genetic relationship and similarity between all nine varieties. The $0 / 1$ data obtained using SSR marker were used to construct a similarity matrix between all nine varieties of wheat using 'UPGMA' subprogram of NTSYS-PC software (Kumar et al., 2016; Hassan Pervaiz et al., 2010) (Fig. $1)$.

The hierarchical cluster analysis revealed that varieties were mainly divided into 5 major clusters (Figure 2). The cluster I is further subdivided into 2 sub clusters. Sub cluster C consist of variety (V3: GW 451) and sub cluster D consist of variety (V1: GW 503). Cluster II comprised of only one variety (V2: GW 496). Cluster III is subdivided into 2 subclusters $\mathrm{A}$ and $\mathrm{B}$ which are further subdivided into E (V8: GW 273) and F (V4: GW 11), G (V9: GW 366) and $\mathrm{H}$ (V6: GW 1255) respectively. Cluster IV and V comprised of only 1 variety (V5: LOC 1) and (V7: GW173) respectively. The dendrogram shows that amongst all the varieties, the most closely related varieties are in cluster III and cluster I. In cluster I, variety V1 (GW503) and V3 (GW451) are closely related to each other. In sub cluster A of cluster III, varieties V4 (GW11) and V8 (GW273) and in sub cluster $\mathrm{B}$, varieties V6 (GW1255) and V9 (GW366) are closely related to each other respectively. While V7 (GW173) and V3 (GW451) are the most distinct varieties among all the 9 varieties. It is noticed that wheat variety
GW173 and GW451 are most diverse variety and used for further breeding programs( $\mathrm{Nei}$ 1972).

\section{Correlation analysis}

The correlation study was carried out to know the similarity between the morphological characteristics of the plant. The results illustrate that $\mathrm{GW}$ is in negative correlation with RL, RDW and SDW, while it is in positive correlation with $\mathrm{ShL}$ and $\mathrm{SpL}$ (Table 2). The RL is seen to have a negative correlation with $\mathrm{ShL}$ and $\mathrm{SpL}$, while it has a positive correlation with RDW and SDW. The $\mathrm{ShL}$ is in negative correlation with RDW and SDW and in positive correlation with SpL. The RDW is in negative correlation with SpLand in positive correlation with SDW. The SDW is in negative correlation with SpL. The positive correlation obtained shows the significance of similarity between the characteristics. This correlation shows that in normal timely sown irrigated conditions there is adequate absorption of water and adequate growth and thus it shows that GW has significant positive correlation with SpL.

The correlation between different varieties was confirmed by descriptive analysis and Pearson Correlation Matrix analysis. With the help of morphological data, the standard deviation was calculated. The Pearson Correlation Matrix was analyzed between the varieties in one cluster(Börner, Chebotar, and Korzun 2000; Hammer et al., 2000, n.d.)

The cluster I is subdivided into 2 subclusters. Sub-cluster C consist of variety (V3: GW $451)$ and subcluster D consist of variety (V1: GW 503). The results illustrate no negative correlation instead shows a positive significant correlation between all the characters. Hence it can be deduced that the two varieties are closely related and have a positive significance. 
Table.1 List of primers used

\begin{tabular}{|l|l|l|l|l|}
\hline \multicolumn{1}{|c|}{ SSR marker } & \multicolumn{1}{|c|}{ Primer Sequence 5' to 3' } & \multicolumn{1}{c|}{$\begin{array}{c}\text { No. of } \\
\text { bases }\end{array}$} & $\begin{array}{c}\text { Chromosomal } \\
\text { position }\end{array}$ & $\begin{array}{c}\text { Product } \\
\text { length }\end{array}$ \\
\hline GWM413 F & TGCTTGTCTAGATTGCTTGGG & 21 & $1 \mathrm{~A}$ & 200 \\
\hline GWM413 R & GATCGTCTCGTCCTTGGCA & 19 & $1 \mathrm{~A}$ & 200 \\
\hline GWM122 F & GGGTGGGAGAAAGGAGATG & 19 & $2 \mathrm{~A}$ & 100 \\
\hline GWM122 R & AAACCATCCTCCATCCTGG & 19 & $2 \mathrm{~A}$ & 100 \\
\hline GWM369 F & CTGCAGGCCATGATGATG & 18 & $3 \mathrm{~A}$ & $200-1000$ \\
\hline GWM369 R & ACCGTGGGTGTTGTGAGC & 18 & $3 \mathrm{~A}$ & $200-1000$ \\
\hline GWM610 F & CTGCCTTCTCCATGGTTTGT & 20 & $4 \mathrm{~A}$ & $100-200$ \\
\hline GWM610 R & AATGGCCAAAGGTTATGAAGG & 21 & $4 \mathrm{~A}$ & $100-200$ \\
\hline GWM570 F & TCGCCTTTTACAGTCGGC & 18 & $6 \mathrm{~A}$ & 100 \\
\hline GWM570 R & ATGGGTAGCTGAGAGCCAAA & 20 & $6 \mathrm{~A}$ & 100 \\
\hline GWM332 F & AGCCAGCAAGTCACCAAAAC & 20 & $7 \mathrm{~A}$ & 200 \\
\hline GWM332 R & AGTGCTGGAAAGAGTAGTGAAGC & 23 & $7 \mathrm{~A}$ & 200 \\
\hline GWM124 F & GCCATGGCTATCACCCAG & 18 & $1 \mathrm{~B}$ & 200 \\
\hline GWM124 R & ACTGTTCGGTGCAATTTGAG & 20 & $1 \mathrm{~B}$ & 200 \\
\hline GWM247 F & GCAATCTTTTTCTGACCACG & 21 & $3 \mathrm{~B}$ & $100-200$ \\
\hline GWM247 R & ATGTGCATGTCGGACGC & 17 & $3 \mathrm{~B}$ & $100-200$ \\
\hline WMC048 F & GAGGGTTCTGAAATGTTTTGCC & 22 & $4 \mathrm{~B}$ & 123 \\
\hline WMC048 R & ACGTGCTAGGGAGGTATCTTGC & 22 & $4 \mathrm{~B}$ & 123 \\
\hline GWM499 F & ACTTGTATGCTCCATTGATTGG & 22 & 5B & 100 \\
\hline GWM499 R & GGGGAGTGGAAACTGCATAA & 20 & 5B & 100 \\
\hline GWM311 F & TCACGTGGAAGACGCTCC & 18 & $6 \mathrm{~B}$ & 100 \\
\hline GWM311 R & CTACGTGCACCACCATTTTG & 20 & $6 \mathrm{~B}$ & 100 \\
\hline GWM437 F & GATCAAGACTTTTGTATCTCTC & 22 & $7 \mathrm{D}$ & $100-160$ \\
\hline GWM437 R & GATGTCCAACAGTTAGCTTA & 20 & $7 \mathrm{D}$ & $100-160$ \\
\hline WMC089 F & ATGTCCACGTGCTAGGGAGGTA & 22 & $4 \mathrm{~B}$ & $120-500$ \\
\hline WMC089 R & TTGCCTCCCAAGACGAAATAAC & 22 & $4 \mathrm{~B}$ & 180 \\
\hline GWM428 F & CGAGGCAGCGAGGATTT & 17 & $1 \mathrm{~B}$ & 1800 \\
\hline GWM428 R & TTCTCCACTAGCCCCGC & 17 & $1 \mathrm{~B}$ & \\
\hline & & & & 180 \\
\hline
\end{tabular}

Table.2 Correlation analysis of morphological characters of wheat. It shows the correlation between six different variables: Grain weight (GW), Root length (RL), Shoot length (ShL), Root dry weight (RDW), Shoot dry weight (SDW) and Spike length (SpL)

\begin{tabular}{|l|l|l|l|l|l|l|}
\hline $\begin{array}{l}\text { Pearson } \\
\text { correlation } \\
\text { matrix }\end{array}$ & GW & RL & ShL & RDW & SDW & SpL \\
\hline GW & 1 & & & & & \\
\hline RL & $-0.451^{\mathrm{NS}}$ & 1 & & & \\
\hline ShL & $0.621^{\mathrm{NS}}$ & $-0.245^{\mathrm{NS}}$ & 1 & & \\
\hline RDW & $-0.501^{\mathrm{NS}}$ & $0.885^{* *}$ & $-0.188^{\mathrm{NS}}$ & 1 & \\
\hline SDW & $-0.483^{\mathrm{NS}}$ & $0.536^{\mathrm{NS}}$ & $-0.084^{\mathrm{NS}}$ & $0.827^{* *}$ & 1 & \\
\hline SpL & $0.859^{* *}$ & $-0.691^{*}$ & $0.463^{\mathrm{NS}}$ & $-0.809^{* *}$ & $-0.680^{*}$ & 1 \\
\hline
\end{tabular}

Note "*" $=$ p-value less than or equal to $0.05 ; “ * * "=$-value less than or equal to 0.01 ; "NS" $=$ no significance 
Fig.1 Agarose gel electrophoresis showing DNA banding pattern of different wheat varieties (V1: GW 503, V2: GW 496, V3: GW 451, V4: GW 11, V5: LOC 1, V6: GW 1255, V7: GW 173, V8: GW 273, V9: GW 366) A) Represents monomorphic bands of Marker GWM 124 in 9 varieties. B) Represents polymorphic bands of Marker WMC 089 in 9 varieties. C) Represents monomorphic bands of Marker GWM 499 in 9 varieties. D) Represents monomorphic bands of Marker GWM 332 in 9 varieties

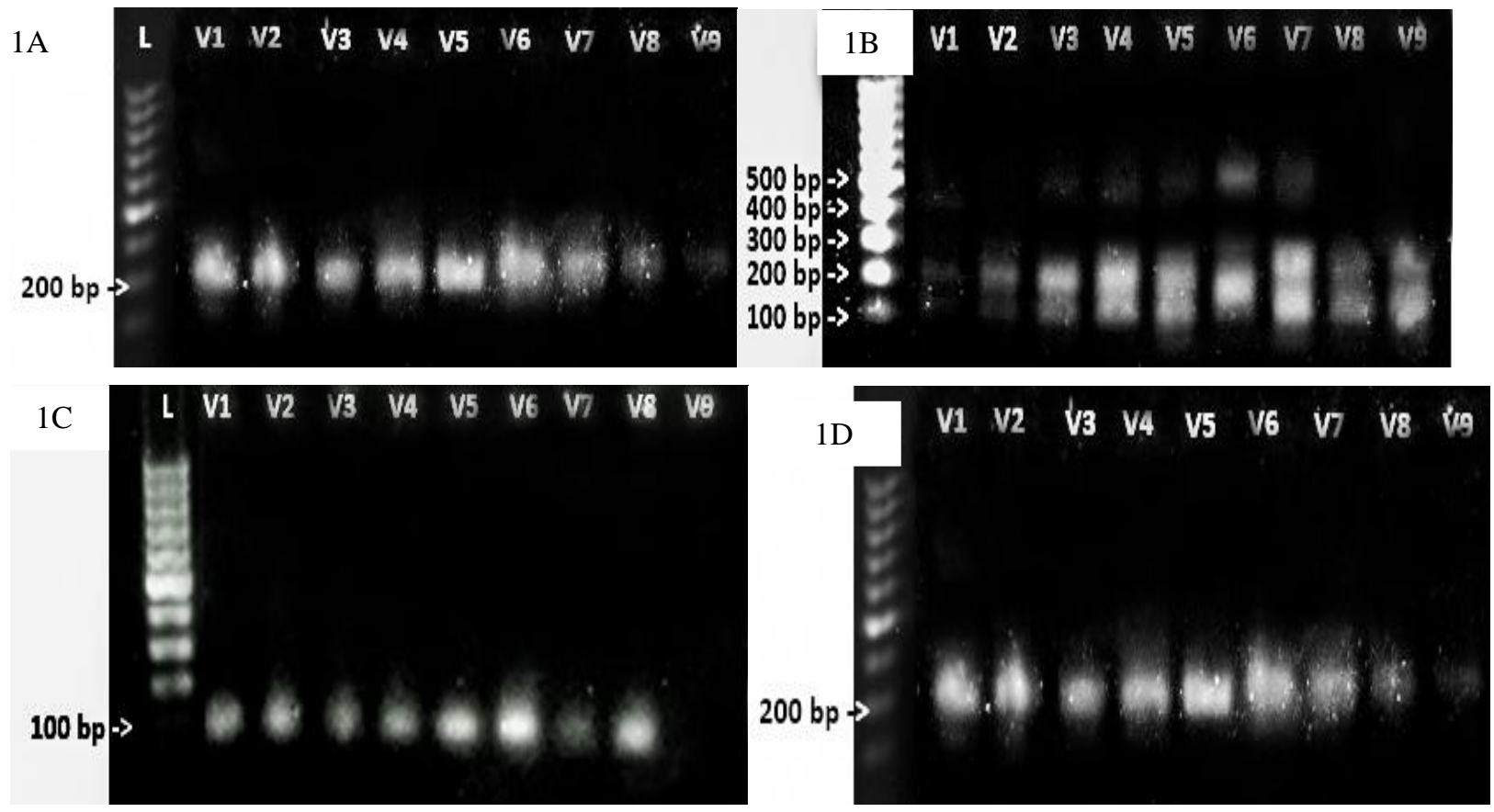

Fig.2 Dendrogram showing the relationship among nine wheat varieties generated by UPGMA analysis

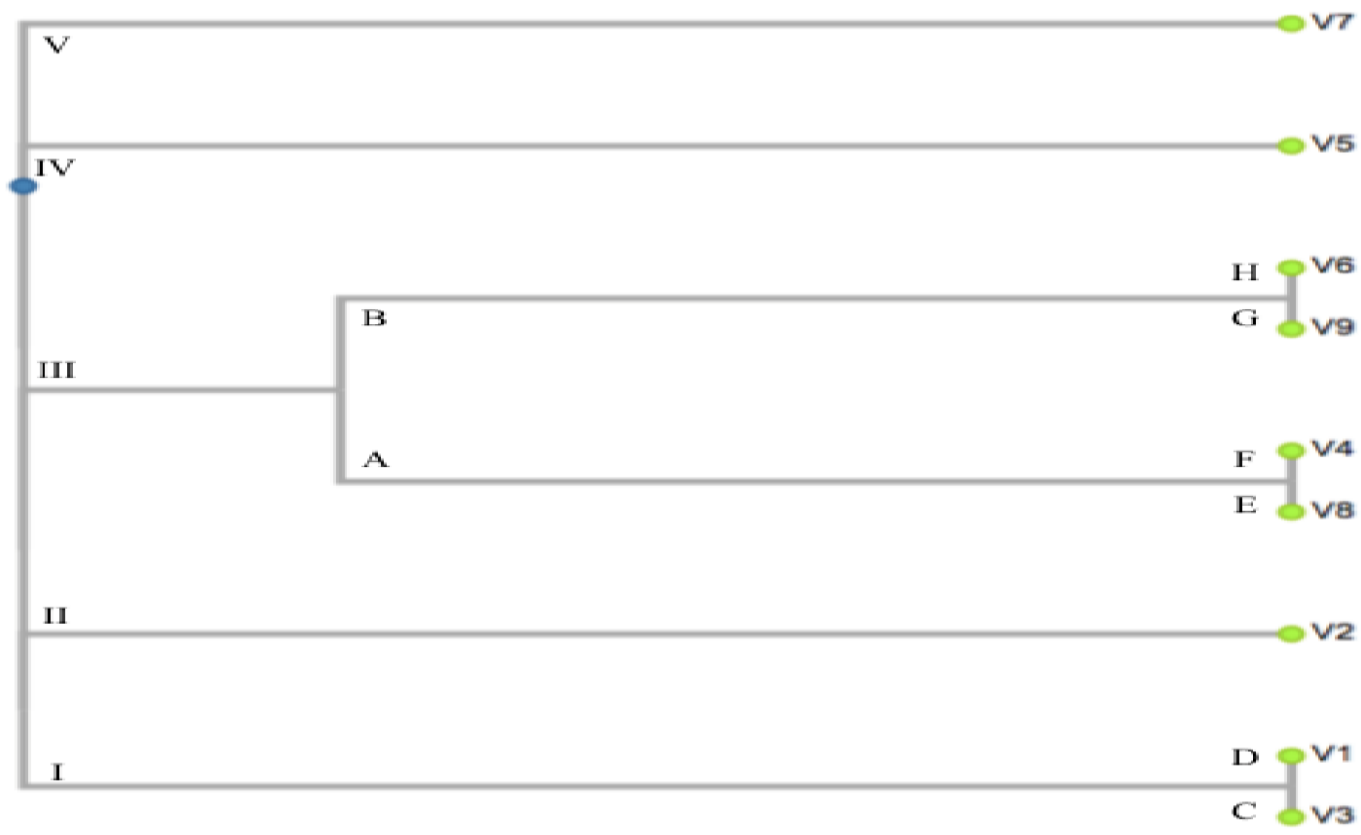


The cluster III consists of (V8: GW 273) and (V4: GW 11) which are closely related to each other. The results show the positive significance of all the characters. The cluster III also consists of (V9: GW 366) and (V6: GW 1255) which are seen to have a close correlation. The correlation is found to be significant in all the characters. Cluster V comprises only one variety (V7: GW173) the sub-cluster $\mathrm{C}$ of cluster I consist of variety (V3: GW 451). These two varieties are the most distant one and hence are found to have the least significance. There is less significant correlation found, however, these varieties do not show negative correlation(Nei 1972). The results of the Pearson Correlation Matrix between the varieties in one cluster confirmed our results of genetic analysis. The Pearson Correlation Matrix confirms that the varieties V6:V9, V4:V8 and V1:V3 are the most closely related varieties respectively. In future, there is a possibility to crossbreed these closely related varieties V6:V9, V4:V8 and V1:V3 for enhancing the dominant characters for better crop productivity. On the other hand distantly related varieties can also be backcrossed for advancement of segregating lines to express some recessive characters.

In conclusion, agriculturists have been realized that diverse plant genetic resources are priceless assets for human kind which cannot be lost. From the result and discussion above, it is concluded that the evaluation of genetic diversity and identification of wheat varieties by AGE is easier and early approach. These could help in improving the efficiency of a wheat breeding program in cultivars development. With the high throughput molecular marker technologies in ensuring speed and quality of data generated, it is possible to characterize a large number of germ-plasm with limited time and resources. From the cluster analysis on the basis of AGE, it was found that wheat varieties V6
(GW1255), V9 (GW366), V4 (GW11) and V8 (GW273) originate from the same cluster III and these varieties are the most closely related varieties. While V7 (GW173) and V3 (GW451) are the most distinct varieties among all the 9 varieties. Also, the morphological analysis data concluded that V6, V9, V4, and V8 are closely related varieties while V7 and V3 are distinct varieties. Hence a possibility of cross breeding of closely or distant related varieties can be a future scope of research and can lead to development of new variety of wheat depending on the specific characters.

\section{References}

Al Khanjari, S., K. Hammer, A. Buerkert, and M. S. Röder. 2007. "Molecular Diversity of Omani Wheat Revealed by Microsatellites: II. Hexaploid Landraces." Genetic Resources and Crop Evolution 54 (7): 140717. https://doi.org/10.1007/s10722-0069125-1.

Arun Gupta, Charan Singh, Vineet Kumar, BsTyagi, Vinod Tiwari, Ravish Chatrath, And Gp Singh. N.D. "Wheat Varieties Notified In India Since 1965." Director ICAR- Indian Institute of Wheat \& Barley Research. http://www.iiwbr.org/wpcontent/uploads/2018/08/wheat-varietiesnotified-in-india.pdf.

Börner, A., S. Chebotar, and V. Korzun. 2000. "Molecular Characterization of the Genetic Integrity of Wheat (Triticum aestivum L.) Germplasm after Long-Term Maintenance:" Theoretical and Applied Genetics 100 (3-4): 494-97. https://doi.org/10.1007/s001220050064.

Hammer et al., 2000. n.d. "Microsatellite Markers - a New Tool for Distinguishing Diploid Wheat Species." Genet. Resour. Crop Evol. 47: 497-505.

Hassan Pervaiz, Zahida, M. Ashiq Rabbani, IshtiaqKhaliq, Stephen R. Pearce, and Salman A. Malik. 2010. "Genetic Diversity Associated with Agronomic Traits Using Microsatellite Markers in Pakistani Rice Landraces." Electronic Journal of 
Biotechnology 13

https://doi.org/10.2225/vol13-issue3-

(3).

fulltext-5.

Ijaz, S., and I. A. Khan. 2009. "Molecular Characterization of Wheat Germplasm Using Microsatellite Markers." Genetics and Molecular Research: GMR 8 (3): 809-15.

Jiang, Guo-Liang. 2013. "Molecular Markers and Marker-Assisted Breeding in Plants." In Plant Breeding from Laboratories to Fields, edited by Sven Bode Andersen. InTech. https://doi.org/10.5772/52583.

Kesawat, Mahipal Singh, and Basanta Das Kumar. 2009. "Molecular Markers: It's Application in Crop Improvement." Journal of Crop Science and Biotechnology 12 (4): 169-81. https://doi.org/10.1007/s12892-0090124-6.

Kumar, Pawan, Ramesh Kumar Yadava, Sandeep Kumar, and Pritam Kumar. 2016. "Molecular Diversity Analysis in Wheat Genotypes Using SSR Markers." Electronic Journal of Plant Breeding 7 (2): 464. https://doi.org/10.5958/0975928X.2016.00060.0.

Lateef, DjshwarDhahir. 2015. "DNA Marker Technologies in Plants and Applications for Crop Improvements." Journal of Biosciences and Medicines 03 (05): 7-18. https://doi.org/10.4236/jbm.2015.35002.

Liu, Jiancheng, Like Liu, NingHou, Aimin Zhang, and Chunguang Liu. 2007. "Genetic Diversity of Wheat Gene Pool of Recurrent Selection Assessed by Microsatellite Markers and Morphological Traits." Euphytica 155 (1-2): 249-58. https://doi.org/10.1007/s10681-006-9326-x.

Nei, Masatoshi. 1972. "Genetic Distance between Populations." The American Naturalist 106 (949): 283-92. https://doi.org/10.1086/282771.

Raj, R. Sandeep, Yama S. Vyas, Viral Kumar M.
Baranda, Madhvi N. Joshi, ShradhaNand Tyagi, and Snehal B. Bagatharia. 2017. "Ascertaining Narrow Genetic Base in Commercial Accessions of Wheat Commonly Grown in Gujarat via Molecular Markers." Electronic Journal of Plant $\begin{array}{lll}\text { Breeding } \quad 8 & \text { (2): } \quad 558 .\end{array}$ https://doi.org/10.5958/0975928X.2017.00084.9.

Saghai-Maroof, M. A., K. M. Soliman, R. A. Jorgensen, and R. W. Allard. 1984. "Ribosomal DNA Spacer-Length Polymorphisms in Barley: Mendelian Inheritance, Chromosomal Location, and Population Dynamics." Proceedings of the National Academy of Sciences of the United States of America 81 (24): 8014-18.

Salem et al., n.d. "Genetic Diversity Using Morphological Characters and Microsatellitete Markers." World J. Agril. Sci. 4 (5): 538-44.

Shewry, P. R. 2009. "Wheat." Journal of Experimental Botany 60 (6): 1537-53. https://doi.org/10.1093/jxb/erp058.

Shuaib et al., 2010. "Evaluation of Wheat by Poly-Acrylamide Gel Electrophoresis, African Journal of Biotechnology Vol. 9 (2) Pp. 243-247, 11 January, 2010." African Journal of Biotechnology Vol. 9 (2) (January): 243-47.

Tomar, Ram Sewak Singh, Sushma Tiwari, Vinod, Bhojaraja K. Naik, Suresh Chand, RupeshDeshmukh, NiharikaMallick, Sanjay Singh, Nagendra Kumar Singh, and S. M. S. Tomar. 2016. "Molecular and MorphoAgronomical Characterization of Root Architecture at Seedling and Reproductive Stages for Drought Tolerance in Wheat." Edited by Rattan Singh Yadav. PLOS ONE 11 (6): e0156528. https://doi.org/10.1371/ journal.pone.0156528.

\section{How to cite this article:}

Summy Yadav, AkdasbanuVijapura, Akanksha Dave, Sneha Shah and ZebaMemon. 2019. Genetic Diversity Analysis of Different Wheat [Triticum aestivum (L.)] Varieties Using SSR Markers. Int.J.Curr.Microbiol.App.Sci. 8(02): 839-846.

doi: https://doi.org/10.20546/ijcmas.2019.802.095 